New techniques

\title{
Percutaneous endoscopic gastrostomy
}

\author{
Anwar Hussain, Sheila Woolfrey, Jane Massey, Ann Geddes, James Cox
}

\begin{abstract}
Summary
Percutaneous endoscopic gastrostomy (PEG) is now the technique of choice for patients requiring long-term enteral feeding. It is a good method for feeding patients with neurological dysphagia and can safely be kept in situ for long periods. PEG feeding requires a multidisciplinary approach, involving doctors, nurses pharmacists, speech therapists, dieticians and carers. The insertion of a PEG, which requires two operators and two endoscopy nurses, is described in full. Feeding can commence after a few hours. Various types of feed and feed-ing patterns are described and their relative merits discussed. Although psychological problems may occur following PEG insertion, the procedure is usually well accepted by patients.
\end{abstract}

Keywords: percutaneous endoscopic gastrostomy, feeding, stroke

\author{
Medicine for the Elderly and General \\ Medicine, Guy's Hospital, London SE1 \\ 9RT, UK \\ A Hussain \\ Cheviot \& Wansbeck NHS Trust, \\ Wansbeck General Hospital, \\ Northumberland NE63 9JJ, UK \\ Clinical Services \\ $S$ Woolfrey \\ Dietetic Services \\ A Geddes \\ Medicine for the Elderly and General \\ Medicine \\ $\mathrm{J}$ Cox \\ Speech \& Language Therapy Unit \\ J Massey
}

Correspondence to Dr JC Cox, Wansbeck General Hospital, Ashington, Northumberland NE63 9JJ, UK

Accepted 3 January 1996
Patients who are malnourished on admission to hospital have longer hospital stays, experience more complications and are at a greater risk of dying than well-nourished patients with a similar illness. ${ }^{1,2}$ Enteral nutrition with fine bore nasogastric tubes has been the mainstay until now, but they are often poorly tolerated and extubation is common. ${ }^{3}$ Percutaneous endoscopic gastrostomy (PEG) is now the technique of choice for longer term administration, replacing surgical gastrostomy. Any patient likely to require enteral feeding for more than four weeks should be considered for PEG.

PEG under local anaesthetic was first described in 1980 for obtaining access to the stomach in patients requiring long-term tube feeding. ${ }^{4}$ Since its first description, PEG has become one of the more favoured techniques of feeding elderly and frail people with persistent swallowing difficulties due to neurological or oropharyngeal disorders. PEG feeding is well tolerated by patients and has a low morbidity $(6-16 \%)$ and mortality $(0-1 \%) .^{5}$

\section{Who should have a PEG?}

We feel that feeding with PEG is a good method for feeding patients with neurological dysphagia. If the patient is being considered for nasogastric or peripheral line feeding, PEG feeding may well be less invasive and more comfortable. Many doctors feel reluctant to use PEG seeing it as keeping severely disabled patients alive when this may not be in their best interests. We feel the decision to be active should come first and then the appropriate method chosen. Peripheral line feeding and nasogastric feeding have their places but the comfort of a PEG over these methods is striking.

We recently audited our use of PEG feeding in patients with dysphagia after stroke. Seventy-five consecutive patients (39 males) with swallowing difficulties after stroke were included. We offered PEG feeding at the end of 7-10 days unless a speech therapy assessment showed return of swallowing to be imminent. Patients remained nil by mouth and received intravenous fluids or peripheral feeding until the insertion of the PEG. The results of this audit showed that those who had difficulty swallowing after 10 days took a surprisingly long time to recover swallowing (median 69 days, range 19405). The PEG tube could be kept in situ for a long time (median 89 days, range $19-600$ ) with safety.

We now routinely use PEG feeding in patients whose swallowing has not recovered by day 10 . Even those whose swallowing has recovered and are allowed to eat orally may not get enough calories, ${ }^{6}$ and may need a PEG for dietary supplementation. We use nasogastric feeding only in those patients who cannot have a PEG inserted and peripheral line feeding only as a temporary holding measure.

\section{Team approach}

The use of PEG demands skilled operators for insertion but more than this, it requires a multidisciplinary approach involving the speech and language therapists, nurses, pharmacists and dietitians, the patients and carers of the patients. Ideally, full discussion and consultation should take place before insertion of a PEG. It is not enough to consider insertion of a PEG as a purely physical manoeuvre performed by a doctor. It is essential that all members of the multidisciplinary team keep up-to-date with the patient's current feeding regimen. This will ensure that a consistent approach to the patient's management is maintained, improving the quality of the care given and facilitating the patient's rehabilitation. Team members will be able to reinforce the reasons for the patient's current regime to the patient and support him/her in carrying out any exercises or swallowing techniques which are necessary. With a clear management plan this process can be facilitated. Adaptation to PEG feeding by the patients and their carers is helped if they understand the reasons for its implementation and if staff reinforce these. Considerable support 


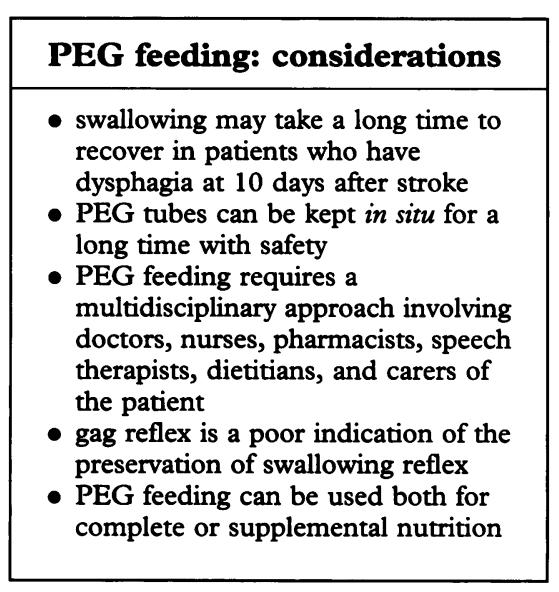

Box 1

\begin{tabular}{|l|}
\hline PEG: contraindications \\
\hline Absolute \\
- gastric outlet obstruction \\
- tense ascites \\
- full anticoagulant therapy \\
Relative \\
- past partial gastrectomy \\
- large intrathoracic hiatus hernia \\
\hline
\end{tabular}

Box 2 is often needed during this time as oral feeding serves important social functions which are lost when feeding is disrupted.

There are some patients whose prognosis is so poor that the insertion of a PEG, though possible, is not in their best interests. Discussion with the patient, carers, and professionals on whether insertion of a PEG is likely to enhance the patient's recovery and quality of life, or alternatively to cause increased suffering, should clearly take place prior to the procedure. It is important to be realistic and yet give the benefit of doubt - it is not always clear beforehand what the right decision is. In general, if there is uncertainty and the patient is already being maintained by a drip or a naso-gastric tube, insertion of a PEG may be the correct decision to buy time while further assessment and treatment of the underlying condition is performed.

\section{Insertion of a PEG}

\section{GENERAL CONSIDERATIONS}

Patients should be carefully screened before PEG insertion for contraindications (box 2). Site selection is important for safe cutaneous penetration. It is necessary to get adequate clearance from the costal margin to avoid discomfort from tube motion due to respiratory excursion. The left lobe of the liver should be palpated in order to avoid its penetration. In patients who have had previous abdominal surgery the possibility of adhesions must be considered. Obese patients with swallowing difficulties may be difficult if the cannula cannot reach the stomach.

\section{PROCEDURE}

We perform PEG insertion using an endoscopic technique in the Endoscopy Unit. Care should be used when using sedation for the procedure as many patients with stroke require less sedation than patients requiring gastroscopy under normal circumstances. We give small amounts and slowly. It is mandatory when sedating a patient with a recent stroke to use pulse oximetry throughout the procedure as such patients may quickly desaturate. We routinely use supplemental oxygen via nasal catheters before and throughout the procedure. It may be necessary and desirable to reverse the sedative with an appropriate antidote after the procedure.

It is commonly thought that oral and dental hygiene is important in terms of inserting a PEG but we have not found this to be the case. Some groups also advocate the discontinuation of $\mathrm{H}_{2}$ blockers and antacids 24 hours prior to PEG but we have not found this necessary. Some groups also advocate routine antibiotics to cover the insertion of PEG; again we have not found this necessary, although, when inserting PEGs into obese diabetics, it may be sensible to use two doses of broad spectrum cephalosporin to avoid cellulitis. Occasionally the cannula passes into the abdomen but does not enter the stomach; under these circumstances we would probably reconsider the entry site and give antibiotics because of a failed attempt. The description that follows is for the insertion of a Freka ${ }^{\mathrm{R}} \mathrm{PEG}$.

Two operators and two endoscopy nurses are required. The first operator, the endoscopist, passes the endoscope by mouth into the stomach. Patients are frequently dehydrated and their lips may be easily traumatized by the endoscope and it is wise to lubricate the lips as well as the endoscope to prevent lip trauma. As the procedure is done with the patient lying flat on his back, it is mandatory to have effective mouth suction available. Sometimes the patient's mouth is full of secretions and suction may be needed prior to the procedure. If the patient's mouth is dry we moisten the tongue and palate with water. The endoscopist inflates the stomach thus allowing the anterior wall of the stomach to meet the anterior abdominal wall. The best site of the proposed gastrostomy tube can then be identified by either transillumination from within by the endoscope or by digital compression of the anterior abdominal wall by the assistant. The choice of site is important and the endoscopist must guide the assistant to the ideal site. This is usually about $2 \mathrm{~cm}$ below the left costal margin and $2 \mathrm{~cm}$ from the midline, although there is considerable variation. From the endoscopist's point of the view, the ideal site is in the anterior stomach wall where the assistant's finger depresses most acutely-it is quite possible to be misled unless selection of the site is rigorous.

When the site has been chosen, it is marked carefully by a small indentationwe use a syringe needle sheath to make this, as this causes a sustained indentation and is better than using ink which washes away with the skin preparation. The assistant who has scrubbed up and has a sterile gown and gloves then cleans the skin. We use a specially made drape with an $8 \mathrm{~cm}$ square hole in its centre and this supplies a satisfactory drape for the whole procedure. 


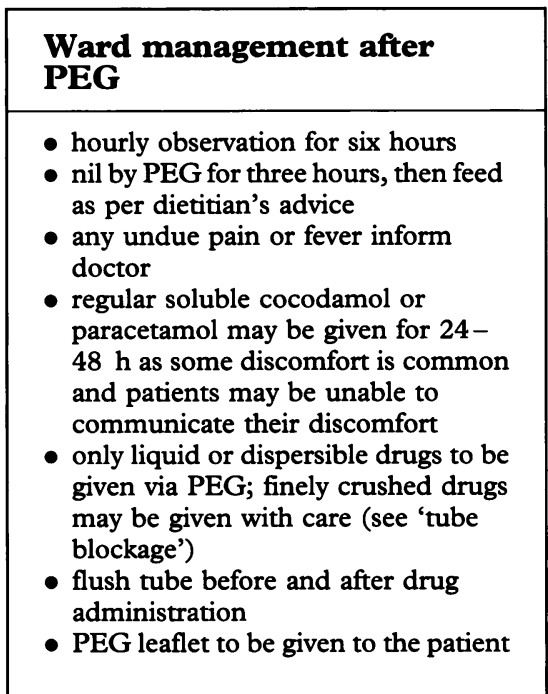

Box 3
The assistant anesthetizes the area with $1 \%$ lignocaine. The use of local anaesthetic is important-midazolam is not an analgesic and the procedure itself can be quite uncomfortable. The assistant infiltrates first with a blue needle ( 23 gauge) to raise a bleb and thereafter with a green needle (21 gauge) directly through the anterior abdominal wall and into the stomach. This acts as a safety measure as the endoscopist can see the needle entering the stomach and know that the site and track selected is suitable. The infiltrated entry site is then incised with a scalpel - we use a pointed $3 \mathrm{~mm}$ width scalpel to make a $3 \mathrm{~mm}$ or $5 \mathrm{~mm}$ diameter incision, depending upon which PEG tube is used. The assistant passes the cannula through the skin into the stomach, removing the inner needle when the operator confirms the cannula is inside the stomach. The plastic outer sheath of the cannula is passed up to its hilt into the stomach and the inner needle removed. A valve is placed at the end of the cannula sheath to prevent air escaping as otherwise the stomach will deflate. A thread is passed through the valve and cannula sheath into the gastric lumen and this thread is grasped by the endoscopist using forceps and drawn through the mouth by removing the endoscope. The assistant must hang on to the other end of the thread. The thread is removed from the forceps and the PEG tube is tied by the endoscopist to the thread; the assistant then pulls the PEG through the mouth into the stomach and back out through the abdominal wall. The endoscope is reinserted to check the position of the PEG. The PEG is then secured by a clip with a firm, but not tight, grip as an overtight compression may lead to gastric wall necrosis. The thread is cut off and the luer attachment for feeding put in place. A final check for excessive tightness is made. A simple dressing is applied over the entry site.

After PEG placement it is our routine practice to keep the patients 'nil by PEG' for three hours and thereafter to give regular soluble cocodamol by PEG for two days since the patients may not communicate the pain of the incision very effectively (box 3 ).

\section{FEEDING}

The enteral feed we use comes in $500 \mathrm{ml}$ glass bottles. Giving sets attach directly on to the bottle which is hung on a drip stand with a plastic harness. One litre plastic bag giving sets are also available but, as the feed has to be decanted, this is more time consuming and there is an increased risk of contamination. The plastic bag giving sets may have a place in home enteral nutrition where the patient is being fed overnight to prevent changing bottles through the night. The bags may also be an advantage for confused patients who may be at risk of disturbing their feed and knocking over the drip stand.

\section{TYPES OF FEED}

Isotonic $(1 \mathrm{Kcal} / \mathrm{ml})$ This is the usual choice to start with. The osmolality of the feed is generally between 280 and $350 \mathrm{mOsm} / \mathrm{kg}$ water.

High energy $(1.5 \mathrm{Kcal} / \mathrm{ml})$ This may be considered if the patient requires a higher energy intake. It may also be used if a shorter feeding time is indicated, but the same energy intake is required. It will usually be introduced once the patient is established on an isotonic feed. The osmolality of the feed is greater than $350 \mathrm{mOsm} / \mathrm{kg}$ water so care is required with the rate of delivery of the feed and ensuring adequate hydration.

High fibre $(1 \mathrm{Kcal} / \mathrm{ml})$ This can be used to help patients with constipation or overflow diarrhoea. The amount used will depend on the individual patient.

Peptide feeds $(1 \mathrm{Kcal} / \mathrm{ml})$ This feed contains partially hydrolysed protein and may be used if there are problems with absorption of the feed or if the patient has not eaten for a long period.

All the above feeds are nutritionally complete and are recommended for PEG feeding. Liquidised, ordinary food is not a desirable alternative because the dilution of food in order to get it into a thin enough consistency to pass through the tube results in a dilution of nutrients and energy, so that the volume required to provide total nutrition is excessive. There would also be an increased risk of bacterial contamination.

\section{ENTERAL FEEDING PUMPS}

We advise the use of enteral feeding pumps as they provide accurate information on the total volume of feed delivered. Once the rate has been set nursing staff can leave the patient to feed knowing that the correct volume will be delivered in the agreed time, thus saving on nursing time. 


\section{CHOICE OF FEEDING PATTERNS}

\section{Continuous feeding}

This is usually recommended at the start of feeding as the patient may not have eaten for several days. This method of feeding will allow the nutritional goal to be reached more quickly. The patient will tolerate the feed better with fewer complications, and is less likely to feel bloated because nutrient absorption is maximized.

If a pump limits the patient's mobility, a portable pump using a bag over the shoulder may be more acceptable. Continuous feeding using feeding pumps may be difficult to manage in confused patients who are likely to pull at the equipment. The rate of feeding is carefully controlled to help prevent problems such as diarrhoea, nausea and vomiting.

\section{Bolus intermittent feeding}

This method of feeding allows set amounts of feed to be delivered in a short period of time, several times a day, eg, $250 \mathrm{ml} / \mathrm{h}$ every four hours. This can be given using an enteral feeding pump or with a syringe. Bolus feeding may cause abdominal distension, and may increase the risk of aspiration. We monitor the patient carefully for aspiration and to ensure that discomfort is not a problem.

\section{Cyclic feeding}

This allows the patient to be fed continuously via a pump over only part of the day, usually $12-16$ hours. A patient on cyclic feeding needs a higher hourly rate of feed than a patient being fed continuously. Careful estimation of the rate of the feed is necessary to ensure the patient tolerates the feed without complications. If the more rapid rate of feeding is not tolerated then cyclic feeding should be given over a longer period of time, eg, 16-18 hours. Cyclic feeding is useful for a patient who is being weaned from PEG feeding. The patient can be PEG-fed overnight and be offered regular oral meals/snacks of appropriate consistency during the day without interference from tube feeding. The amount of feed via the PEG can be gradually reduced and discontinued as normal eating returns.

Cyclic feeding also has advantages for patients who cannot eat at all. It is often better tolerated than bolus feeding and frees the patient from feeding during the day.

For patients who are receiving physiotherapy it is recommended that they have a break from feeding for at least one hour prior to their treatment. This will help to prevent regurgitation of feed. If the PEG is inserted over a weekend, we use a provisional feeding regimen until the next working day (box 4).

\section{Maintaining hydration}

The water used to flush the tube has two important functions; firstly, it helps keep the patient adequately hydrated, and secondly, it helps to prevent the tube from getting blocked. As a general rule, most patients need two litres of fluid per day. The patient's water requirements can also be calculated by allowing $30 \mathrm{ml}$ of water per kilogram of bodyweight per day. Patients who are losing fluid from excessive sweating, rapid breathing, diarrhoea or wound drainage need additional fluid. The amount of water per bottle of feed will be stated on the label and this amount should be subtracted from the total volume per day to show the amount of extra water required. This amount can be syringed through the PEG between feeds.

Older adults may be susceptible to dehydration during PEG feeding. Signs of dehydration are thirst, weight loss, decreased skin turgor, oliguria, postural hypotension or altered level of consciousness. Risk factors are infection, diarrhoea, the use of laxatives, or hyperosmolar feeding formulas. Also patients who receive high-protein feeding formulas without sufficient water can suffer from dehydration, hypernatraemia and hyperchloraemia. We monitor the patient's fluid status and electrolytes closely initially until a stable state is achieved.

\section{Overhydration}

Although it is vital to provide sufficient fluid it is also important not to administer too much at once. If too much is given the patient may complain of a feeling of fullness. Smaller amounts administered more frequently may alleviate this problem.

Rapid weight gain, breathlessness and peripheral oedema suggest overhydration. Careful monitoring of fluid intake and output is essential to avoid over-hydration, especially if fluids are also being given orally. A hyperosmolar feed may help to decrease the total amount of water given to the patient. Once 


\section{Possible monitoring criteria in patients fed by PEG}

- diet charts (ie, how much taken)

- weighing (allowing for oedema when doing so)

- skinfold thickness at the level of the triceps

- mid arm circumference and hip: waist ratio

- estimation of body mass index

- biochemical measures including albumin, haemoglobin, red cell folate and vitamin $\mathrm{C}$

\section{Box 5}

\section{Problems with PEG feeding} and their solutions

- pain at PEG site: analgesics via PEG

- infection at PEG site: antibiotics via PEG

- nectrotising fasciitis: early recognition, surgical debridement and antibiotics

- aspiration: if history of previous aspiration place a jejunostomy PEG tube

- hypernatraemia: usually due to inadequate water supplementation

- regurgitation: slow the delivery of feed

- constipation: increase fibre content of feed

- diarrhoea: may be caused by bolus administration, high osmolar feeds, bacterial contamination, or drug therapy

- tube blockage: regular flushing with water; avoid crushed drugs and high viscosity feeds

\section{Box 6}

the PEG is inserted, daily monitoring of PEG feeding is necessary. Monitoring criteria such as those given in box 5 may be helpful but much is done by simple clinical observation. Box 6 describes the various problems associated with PEG feeding and their solutions.

\section{Psychological problems following PEG use}

\section{THE PATIENT}

Patients need help in making the psychological adjustments necessary to accept PEG feeding. This is facilitated by a clear explanation of the reasons for PEG feeding and a discussion of the possible long-term outcomes.

The social importance of eating must not be underestimated, neither should the team forget how the patient needs to cope with the loss of function that PEG feeding can symbolize. As far as possible our policy is to discuss the potential problems with the patient and carer before insertion, especially those who may never have the PEG removed, for example, a patient with motor neuron disease. Most patients and carers, however, cannot conceive of this being a problem prior to insertion of PEG when survival is often the main issue and it is only once they have started on 'a PEG existence' that it dawns on the patient and carer how important it is to gratify the desire to eat. It may be hard for patients to watch others eating when they cannot.

As most of us organise our day around periods before or after a meal it is easy to see how this may lead to patients becoming depressed. Mealtimes are also a time when patients receive attention from ward staff or carers. Some patients who do not get food orally at mealtimes may feel that they are not receiving attention. Clearly counselling is important to prevent patients with PEGs from feeling deprived or depressed. Perhaps surprisingly, despite such problems, we found that the procedure is generally very well accepted by patients.

\section{CARERS}

It is important for the multidisciplinary team to be involved with the carers from an early stage. In practice, the nurses are most intimately involved with the carers but all members of the team play a part. The carers need an explanation of the reasoning for a PEG prior to the procedure and support and instructions on the use of the tube when it comes to discharge. Usually it is wise to involve the carers in the day-to-day running of the PEG tube early in the patient's stay so that it does not become unduly frightening, and they can see that the management is relatively simple.

It is essential that psychosocial support be provided and that plans for discharge are carefully prepared with documented teaching and early and full liaison with the primary healthcare team. Carers can easily become disillusioned and dissatisfied when a lack of knowledge prevents the early solution of simple problems such as tube blockage. A contact number to get urgent advice is essential and each member of the multidisciplinary team must accept an ongoing responsibility towards patients who have had PEGs inserted.

The authors would like to acknowledge all the staff at the Cheviot and Wansbeck Trust who have given advice in the preparation of this article and Miss Susan Smith, Postgraduate Administrator, for her secretarial help.

3 Rees RGP, Payne-James JJ, King C, Silk DBA Spontaneous transpyloric passage and performance of inbore polyurethane feeding tubes: controlled clinical trial. 7 Parenter Enter Nut 1988; 12: 469-72.

4 Gauderer MWL, Ponsky IL, Izant RJ. Gastrostomy without laparotomy: a percutaneous endoscopic technique for feeding gastrostomy. Pediatr Surg 1980; 15: 872-5.
5 Larson DE, Burton DD, Schroeder KW, D Magno EP. Percutaneous endoscopic gastrostomy. Indications, success, complications, and mortality in 314 consecutive patients. Gastroenterology 1987; 93: 48-52.

6 Hussain A, Cox JGC Nutritional intake in patients admitted with acute stroke. Gut 1995; 36: F272. 